\title{
ФУНКЦИЯ ПАМЯТИ ЭКСИТОНОВ ФРЕНКЕЛЯ
}

\author{
F. SOKOLOV. FRENKELI EKSITONIDE MALUFUNKTSIOON \\ F. SOKOLOV. MEMORY FUNCTION OF FRENKEL EXCITONS
}

Формализм обобщенного кинетического уравнения (ОКУ) является общим подходом к различным проблемам неравновесной статистической физики. В. Кенкре и Р. Нокс $\left[{ }^{1}\right]$ предложили использовать ОКУ в теории переноса возбуждений в кристалле и рассмотрели процедуру нахождения функции памяти (ФП) для процесса миграции экситонов. В частности, было получено выражение для ФП через оптические спектры $\left[{ }^{1}\right]$ и проведены расчеты ФП в рамках конкретной модели экситонфононного взаимодействия $\left[{ }^{2}\right]$. В $\left[{ }^{3}\right]$ были уточнены результаты $\left[{ }^{2}\right]$ и получено затухание когерентной части ФП. В отмеченных работах при нахождении ФП в той или иной форме использовалась теория возмущений. Однако для более полного исследования свойств памяти в электронной системе представляет интерес нахождение ФП в рамках таких моделей, которые позволяют сделать это точно,

В данной работе получено точное выражение для ФП в модели, рассмотренной Р. Меррифилдом [ $\left.{ }^{4}\right]$ в связи с изучением эволюции электронного возбуждения в кристалле. В этой модели предполагается ортогональность узельных волновых функций и используется приближение взаимодействия ближайших соседей; не учитывается влияние колебаний решетки на процесс распространения возбуждения. Здесь, кроме того, мы ограничимся рассмотрением случая линейной цепочки молекул.

Если в начальный момент времени $(t=0)$ матрица плотности диагональна в представлении узельных функций $\mid n>(\mid n>-$ состояние с возбуждением на $n$-м узле), то вероятность нахождения возбуждения в момент времени $t$ на $n$-м узле $P_{n}(t)$ удовлетворяет следующему ОКУ $\left[{ }^{1}\right]$ :

$$
\frac{\partial P_{n}(t)}{\partial t}=\int_{0}^{t} d \tau \sum_{m} W_{n m}(t-\tau)\left[P_{m}(\tau)-P_{n}(\tau)\right]
$$

где $W_{n m}(\tau)$ - ФП, для нахождения которой можно использовать точную формулу [5]

$$
W_{n m}(\tau)=-\left[L_{1} \mathrm{e}^{-i \tau(1-D) L} L_{1}\right]_{n n m m}
$$


Здесь тетрадные операторы $L$ и $L_{1}$ представляют собой соответственно оператор Лиувилля и его часть, связанную с гамильтонианом взаимодействия; $D$ - проекционный оператор, выделяющий диагональные элементы матрицы плотности. Однако в рассматриваемой нами задаче для нахождения ФП проще воспользоваться тем, что вид функции $P_{n}(t)$ хорошо известен $\left[{ }^{4}\right]$ и. без решения ОКУ. Если $P_{n}(0)=\delta_{n 0}\left(\delta_{n 0}\right.$ символ Кронекера), то

$$
P_{n}(t)=J_{n}^{2}(b)
$$

где $b=2 \lambda t, \lambda-$ матричный элемент резонансного взаимодействия между ближайшими соседями, $J_{n}(b)$ - функция Бесселя первого рода $n$-го порядка.

Разложим $P_{n}(t)$ в ряд Фурье

$$
P_{n}(t)=\frac{1}{N} \sum_{k} P(k ; t) \cos (k n) .
$$

Здесь $N$ - число узлов, $k$ определяется из периодических граничных условий. Переходя к пределу $N \rightarrow \infty$ и используя (3), а также известную из теории функций Бесселя теорему сложения $\left[{ }^{6}\right]$, получаем

$$
P(k ; t)=J_{0}(2 b \sin k / 2) .
$$

Учтем, что ФП зависит лишь от расстояния между узлами: $W_{n m}(\tau) \equiv W_{g}(\tau) ; g=n-m$. Тогда ОКУ для $P(k ; t)$ принимает вид

$$
\frac{\partial P(k ; t)}{\partial t}=\int_{0}^{t} d \tau W(k ; t-\tau) P(k ; \tau),
$$

где

$$
W(k ; \tau)=\sum_{g} W_{g}(\tau) \cos (k g)
$$

(в (7) учтено правило сумм $\left.\left[{ }^{5}\right]: \quad \sum_{\mathrm{g}} W_{\mathrm{g}}(\tau)=0\right)$.

Используя далее теорему об образе Лапласа свертки, а также известные образы Лапласа функций Бесселя, получаем из (5) и (6) для образа Лапласа

$$
W(k ; p)=\int_{0}^{\infty} d \tau \mathrm{e}^{-p \tau} W(k ; \tau)
$$

следующее выражение:

$$
W(k ; p)=-\frac{16 \lambda^{2} \sin ^{2} k / 2}{p+\sqrt{p^{2}+16 \lambda^{2} \sin ^{2} k / 2}} .
$$

По таблицам (см., напр., $\left.\left[{ }^{7}\right]\right)$ находим соответствующий оригинал преобразования Лапласа

$$
W(k ; \tau)=-2 \lambda^{2} \sin ^{2} k / 2\left[J_{0}(2 b \sin k / 2)+J_{2}(2 b \sin k / 2)\right] .
$$

Обращая далее преобразование Фурье (7), переходя от суммирования по $k$ к интегрированию и используя табличный интеграл [ $\left.{ }^{6}\right]$ 

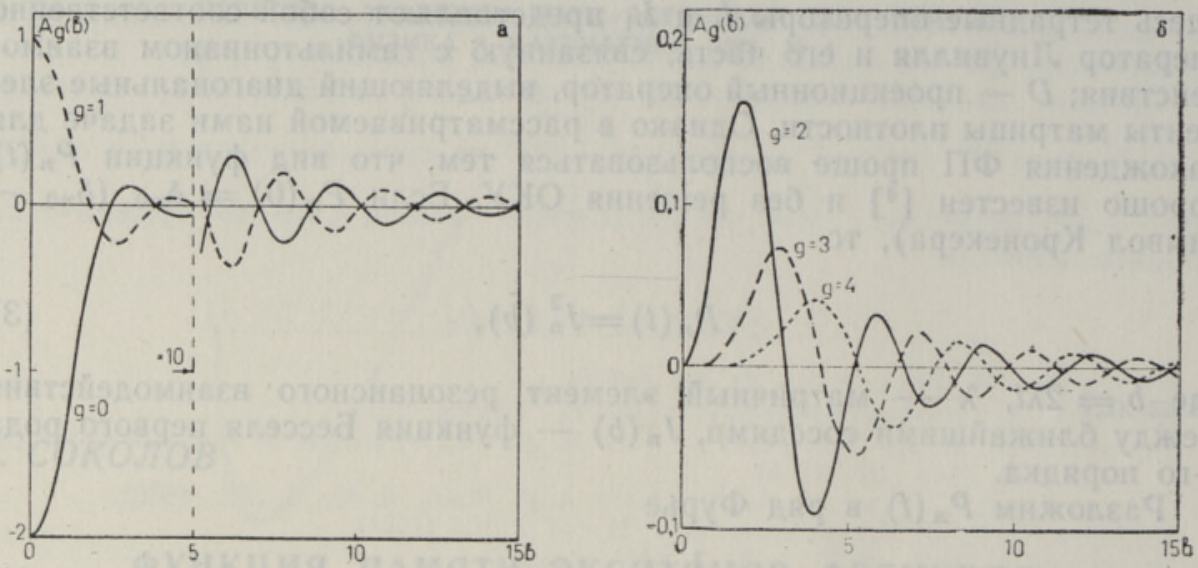

Функция $A_{g}(b)=W_{g}(\tau) / 2 \lambda^{2}$ для $g=0$ и 1 (a) и для $g=2-4$ (б).

$$
\int_{0}^{\pi} d x \cos (2 \mu x) J_{2 v}(2 a \sin x)=\pi \cos (\mu \pi) J_{v-\mu}(a) J_{v+\mu}(a),
$$

получаем окончательно

$$
\begin{gathered}
W_{g}(\tau)=2 \lambda^{2}\left[J_{g-1}^{2}(b)+J_{g+1}^{2}(b)-2 J_{g}^{2}(b)+2 J_{g-1}(b) J_{g+1}(b)-\right. \\
\left.-J_{g}(b) J_{g+2}(b)-J_{g}(b) J_{g-2}(b)\right] .
\end{gathered}
$$

Графики ФП (без множителя $2 \lambda^{2}$ ) для нескольких значений $g$ приведены на рисунке. При достаточно больших $\tau$ ФП имеет асимптотику

$$
W_{g}^{a c}(\tau)=\frac{2}{\pi \tau^{2}} \sin (2 b-\pi g+\pi / 2)
$$

Следует отметить, что найденная в приближении взаимодействия ближайших соседей ФП не равна тождественно нулю и при $|g|>1$. Полученная ФП осциллирует и затухает со временем. Таким образом, в процессе распространения френкелевских электронных возбуждений в кристалле память затухает даже в том случае, когда экситонные состояния с определенными волновыми векторами являются стационарными.

Автор благодарен В. В. Хижнякову за обсуждение результатов и Г. С. Завту за помощь в численных расчетах.

\section{Л И Т Е РА Т У РА}

1. Kenkre V. M., Knox R. S., Phys. Rev., B9, 5279 (1974).

2. Kenkre V., Rahman T., Phys. Lett., A50, 170 (1974).

3. Sokolov F. F., Hizhnyakov V. V., Phys. stat. sol., 75, 669 (1976).

4. Merrifield R. E., J. Chem. Phys., 58, 647 (1958).

5. Z w a n zig R., Physica, 30, 1109 (1964).

6. Г радш тей н И. С., Р ы жик И. М., Таблицы интегралов, сумм, рядов и произведеннй, М., 1963.

7. Д и ткин В. А., Прудн ико в А. П., Справочник по операционному исчислению, M., 1965.

Ннститут физики

Академии наук Эстонской ССР
Поступила в редакцию 17/III 1976 